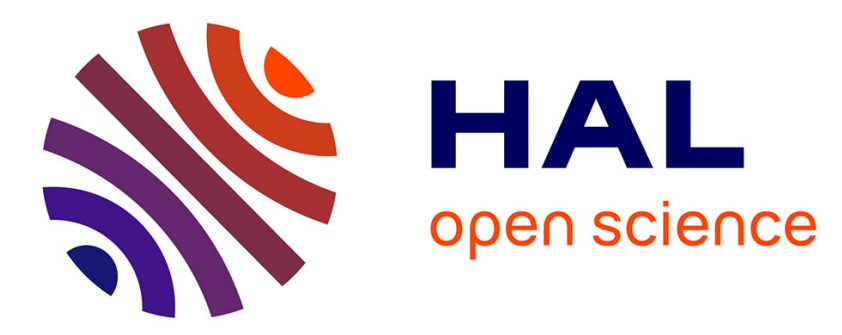

\title{
Direct determination of a single battery internal resistances distribution using a heterogeneous model
}

Maxime Juston, Nicolas Damay, Christophe Forgez, Guy Friedrich, Stephane Vivier, Karrick Mergo Mbeya, Bogdan Vulturescu

\section{- To cite this version:}

Maxime Juston, Nicolas Damay, Christophe Forgez, Guy Friedrich, Stephane Vivier, et al.. Direct determination of a single battery internal resistances distribution using a heterogeneous model. Mathematics and Computers in Simulation, 2020, 10.1016/j.matcom.2020.01.015 . hal-02489055

\section{HAL Id: hal-02489055 \\ https://hal.science/hal-02489055}

Submitted on 24 Feb 2020

HAL is a multi-disciplinary open access archive for the deposit and dissemination of scientific research documents, whether they are published or not. The documents may come from teaching and research institutions in France or abroad, or from public or private research centers.
L'archive ouverte pluridisciplinaire HAL, est destinée au dépôt et à la diffusion de documents scientifiques de niveau recherche, publiés ou non, émanant des établissements d'enseignement et de recherche français ou étrangers, des laboratoires publics ou privés. 


\section{Graphical Abstract}

Direct determination of a single battery internal resistances distribution using a heterogeneous model

Maxime Juston ${ }^{a, b}$, Nicolas Damay $^{a}$, Christophe Forgez $^{a}$, Guy Friedrich ${ }^{a}$, Stephane Vivier ${ }^{a}$, Karrick Mergo Mbeya $^{a}$, Bogdan Vulturescu ${ }^{b}$

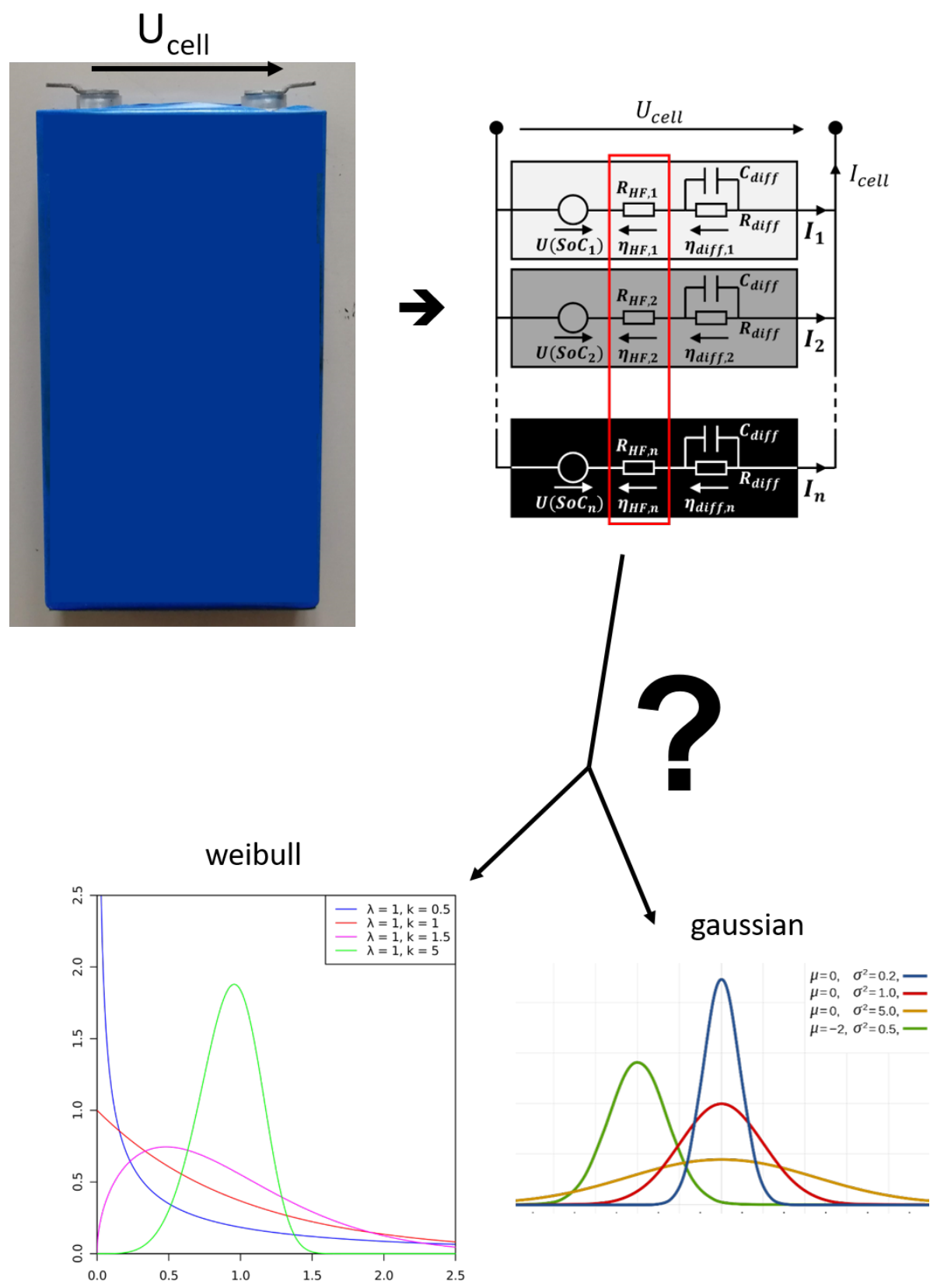




\title{
Direct determination of a single battery internal resistances distribution using a heterogeneous model
}

\author{
Maxime Juston ${ }^{a, b}$, Nicolas Damay $^{a}$, Christophe Forgez $^{a}$, Guy Friedrich $^{a}$, Stephane Vivier $^{a}$, Karrick Mergo Mbeya $^{a}{ }$ \\ Bogdan Vulturescu ${ }^{b}$ \\ (a) Sorbonne Université, Université de technologie de Compiègne, CNRS, FRE 2012 Roberval, Centre de recherche Royallieu, CS 60203 \\ Compiègne Cedex, France \\ (b) SNCF Innovation et Recherche, département TECH4RAIL, 1/3 avenue François Mitterrand, 93212 La Plaine Saint-Denis Cedex, France
}

\begin{abstract}
Lithium-ion batteries are getting larger due to the expansion of transportation and mass storage markets and they can now contain up to thousands of cells. However, a sole damaged cell can significantly impact the whole battery pack efficiency. Thus, the diagnosis of a single cell remains critical for those systems. Many methods exist in which the cell is considered homogeneous. We recently developed a heterogeneous equivalent circuit model that considers an internal resistance distribution to better represent a real single cell behaviour. This internal resistance distribution (IRD) may bring valuable information about a single cell internal quality, but only if it is determined with a sufficient accuracy. In this paper, we propose an algorithm that allows a responsive determination of the IRD. The results are compared to previous determination methods. This IRD, which is determined thanks to the preliminary construction of a homogenous model and a single discharge, is also valid for other operating conditions. The determination of a cell IRD can be used as a non-invasive diagnosis tool to track the internal degradations of a cell. The IRD of two different cell, one aged and one new are then compared, the IRD of the aged one being on average larger. This proves the relevance of the determination method and its use to get an insight into a cell. Differences in shape between the aged and the new cell IRD are discussed, as well as criteria that seems interesting. Although this work is developed for a single cell, its initial goal is to be used to detect a damaged cell inside a battery pack and may thus be applied to several cells connected in parallel.
\end{abstract}

Keywords: battery, heterogeneous electrical model, internal resistance distribution, non-invasive diagnosis

\section{Introduction}

Lithium-ion batteries are becoming one of the best solution to store energy in a wide range of applications, in particular among systems where weight or volume are major constraints, such as drones, cars and trains. In the industry, this technology is not often well known and it is to our advantage to develop a plain, yet robust model, that can be used in a wide range of operating conditions. In order to anticipate the voltage response of a cell to a current profile, a model can be used. Three main types of models exist, with a first one being the electrochemical model. It takes into account the chemical reactions, diffusion reactions and energy equations at the atom scale. Example of this model can be found in many articles such as in Di Domenico et al. (2010) where the author use an electrochemical-based model coupled to a Kalman filter to estimate a cell state

Preprint submitted to MATCOM of charge during usage, or in Yuan et al. (2017) where the authors estimate the lithium concentration within the electrode. In Dong et al. (2018) the authors use an electrochemical model to simulate the thermal behaviour of a cell under very high current charge or discharge, that is more than 8 C. Finally, Delacourt (2016) presents the construction of an electrochemical model. The interested reader will find in this long document all the information regarding the electrochemical modelling of a cell. However, the complexity of those models and the number of parameters needed require invasive measurements that are not possible for most battery managers.

Another main type of model is the black box model that uses mathematical description of a cell using probability and large data set to be constructed. Example of this model can be found in Saha et al. (2009) where the authors estimate the remaining life of a cell using a

February 24, 2020 
Bayesian framework or in Nuhic et al. (2013) where the author use machine learning to estimate the cell remaining life as well. Those models can be built with various methodologies (machine learning, fuzzy logic, genetic algorithms) but have often in common the fact that they are constructed thanks to a large set of data. The result is "black box" model with no or very few physical meaning, which cannot be used to diagnose the internal state of the battery.

One last option in term of model is to use electrical equivalent circuits, also known as behavioural models. Those model are generally done at the cell level and model it thanks to usual electrical components, that is resistances, capacitances, voltage sources, etc. Even though they do not take into account all the atom-level reactions, a good accuracy can be obtained. The interested reader can find more information in the article of Berrueta et al. Berrueta et al. (2018) where the modelling of electrochemical phenomena using an electrical framework is explained. Articles Andre et al. (2011) and Kuhn et al. (2006) focuses on the modelling of unusual electrical component, that are constant phase element and Warburg impedance in RC circuits. Finally, in the article Merla et al. (2018) introduces a multi-particle, diagnosis oriented model. Although those behavioural models are easier to parametrize, their parameters have to depend on current, temperature and State of Charge (SoC) to bring accurate results. Thus, those models usually require lookup tables that need many measurements to be completed.

All those models are useful to predict the performances of a cell in many operating conditions in order to optimize the sizing of a battery pack, choose an appropriate cooling system or predict its capacity to fulfil a mission. A previous work Kuhn et al. (2006) led to a homogeneous equivalent circuit of a Ni-MH cell, built on a physical basis. This model only takes into account the main phenomena that occur inside a cell, and associate to them a combination of resistances and capacitances to model those phenomena.

Recently, the homogeneous model developed in our laboratory was extended to a heterogeneous one, the socalled "multibunch model", by Damay et al. Damay et al. (2017). It aims to model the heterogeneity of a cell thanks to a distribution of one or more parameters. The origin of those distributed parameters can have many sources that will be listed. This model is more accurate than a homogeneous one and although it may appear more complicated at first sight, it is faster to characterize than a usual electrical equivalent circuit model.
Damay et al. found that the addition of the heterogeneous behaviour representation in the model allows the removal of the parameters SoC-dependencies (except for the open circuit voltage). This led to model that is faster to characterize: around 10 times faster than the previous homogeneous equivalent circuit model, which had a SoC-dependency precision of $10 \%$. Once characterized, this model is able to simulate the cell over its whole range of operation. The characterization of the heterogeneity of a cell, through the distribution of one or more parameters, also brings valuable insights into its behaviour and its internal electrical properties. We expect this distribution to change during the cell lifetime due to ageing and the tracking of this distribution may be used as a diagnosis tool.

In this paper, we demonstrate that the measurement of a single discharge, used to build our model, allows us to simulate the cell in 4 different operating conditions and to extract the distribution of one of our parameters. For this article, this parameter is a single resistance modelling fast-dynamic phenomena such as charge transfer, current-collector resistance and electrolyte resistance. The distribution of this resistance for two different cells, one aged and one new, will be computed and compared in order to support the idea that this tool can be used as a non-invasive diagnosis tool. The problems related to the determination of this distribution will be presented and our solutions explained. In section 2 we present the construction of the model, from a homogeneous one to a heterogeneous one, its parameters and variables. The simulation and optimization algorithms are presented in section 3 while section 4 describes our experimental setup and the used cells. Discussions about the obtained results are in section 5 and last, the conclusion and perspectives of this study are presented in section 6 .

\section{Model and parameter dependency}

\subsection{Construction of the multibunch model}

Our model is based on the assumption that one cell can be divided into elementary volumes that behave homogeneously and have specific electrical properties. Those volumes can be seen as paths that are used by electrons and lithium ion to travel through the cell internal parts. Those volumes are connected in parallel, as showed on figure 1.a. We assume that certain volumes, not necessarily spatially bounded, have close electrical properties when compared to each other. Because their behaviours are comparable, we group those volumes into a "bunch" that is considered homogeneous. By repeating this operation, the cell is 

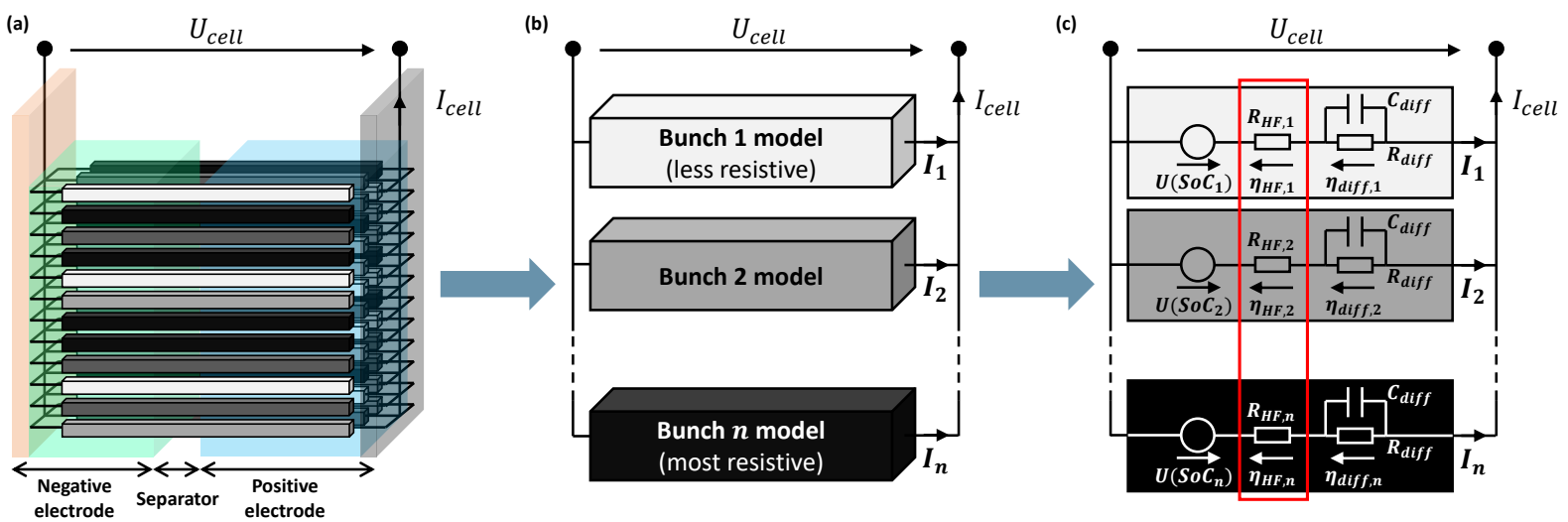

Figure 1: (a) Division of the cell into elementary homogeneous volumes (b) Model with homogeneous volumes grouped (c) Multibunch model with $\mathrm{n}$ bunches

discretized into $n$ homogeneous bunches, each having distinct electrical properties, see figure 1.b. The origin of those distinct electrical properties can be: the position of the tab within the cell Zhao et al. (2014), the distance of active material to current collector Bernardi and Go (2011), temperature gradient, manufacturing non uniformities Schuster et al. (2015), non-uniform pressure on the electrodes Ouvrard et al. (2013), local tortuosity Cooper et al. (2014), different particles sizes Satyavani et al. (2016), etc... This idea of variation of electrical parameters within the cell is supported by the recent work of Park et al. Park et al. (2018), who found that a resistance distribution is found by scanning the surface of an active material sample. The number of bunches of our model, $n$, is a choice from the user and its value is discussed later in this section.

The bunches have a proper current $I_{i}$ but the same voltage: $V_{\text {cell }}$. The sum of the bunch currents $I_{i}$ is the cell current: $I_{\text {cell }}$. Each bunch is modelled using an equivalent circuit model (ECM). The proposed representation of a bunch comes from a previous article from Damay et al. Damay et al. (2015) in which the hypothesises are the following:

- The double layer capacity is neglected because of its fast dynamic and the fact that our tests are conducted at constant current during thirty to sixty minutes. We call "high frequency" resistance, noted $R_{H F}$, the sum of the so called ohmic resistance and the charge transfer one.

- In order to reduce the computation time, the Warburg impedance representing the diffusion phenomenon is modelled by a single RC. More RC circuits can be added to better model the diffusion, as presented in the studies of Kuhn et al. Kuhn et al. (2006) where the transformation from a Warburg element into an infinite sum of RC is explained, and of Andre et al. Andre et al. (2011) where the modelling of a constant phase element with RC circuits is explained. The subscript $d$ under those elements stands for diffusion. This hypothesis is assumed reasonable as the errors in constant current discharge will be concentrated during the first tens of seconds for simulation of several tens of minutes.

Those two hypotheses lead us to the multibunch dynamic model used in this paper, presented on figure 1c: one voltage source, one high frequency resistance and one parallel RC. Because each bunch represent elementary parts with different electrical properties, the value of the resistance will be different from one bunch to another. Among those parameters and to simplify our model, all the heterogeneity of the electrical properties is considered to be concentrated in the values of the $R_{H F, i}$. We expect this parameter to be the most heterogeneous one in a cell due to varying distance to the current collector and variation in the manufacturing processes. The determination of the distribution of the $R_{H F, i}$ is done either by searching a mathematical distribution (a Weibull one), or by searching directly values fitting the experiments. Differences between both methods will be discussed later

\subsection{Parameters values, dependencies and number of bunches}

The voltage source associated to bunch number $i$ has a values $U\left(Q_{i}\right)$ that varies with the bunch charge like an increasing and nonlinear function, following the open 
circuit voltage (OCV) of the cell. The bunch charge is defined by its initial state and the bunch current by

$$
Q_{i, t}=Q_{i, t=0}+\int_{0}^{t} I_{i}(t) \mathrm{d} t
$$

Thus the value of the bunch voltage source $U\left(Q_{i}\right)$ only depends on the proper SoC of the bunch. All others parameters have values that vary with the temperature and the current. The lookup tables we use to represent those variations are obtained through the analysis of the voltage response of the cell to a current pulse. By using different current values at different temperatures, one can extract the variation of the parameters through the analysis of the response, as can be seen on figure 2 . The interested reader will found detailed protocol in the article from Damay et al Damay et al. (2017).

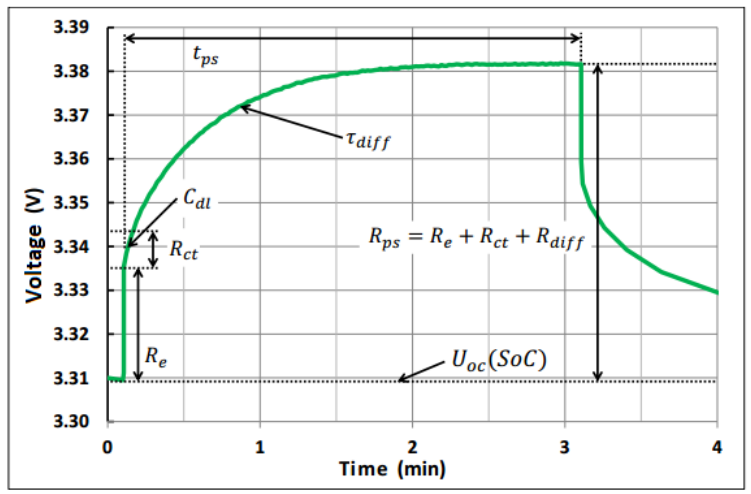

Figure 2: Expected voltage response of a cell

Values of the parameters at different temperatures and currents are then computed as follow, where $\mathrm{P}$ represents a parameter and $\beta_{P(I, T)}$ is a coefficient which is calculated thanks to measurements:

$$
P(I, T)=P\left(50 \% S o C, 1 C, 25^{\circ} C\right) \cdot \beta_{P}(I, T)
$$

where

$$
\beta_{P}(I, T)=\frac{P_{\text {meas }}(50 \% \text { SoC }, I, T)}{P_{\text {meas }}\left(50 \% S o C, 1 C, 25^{\circ} C\right)}
$$

Each parameter has its own non-linearity look-up tables regarding the temperature and the current. If no measurement for the particular I, T does exist, a linear interpolation between the closest measured values is done. Conversely to the $R_{H F, i}$ values, the values of $R_{d}$ and $C_{d}$ are the same for each bunch. Thus no subscript $i$ is added to their names. The distribution of the parameters $R_{H F, i}$ is done by optimization and will be discussed in section 3.2

A summary of the differences between a usual equivalent electrical model and the multibunch model can be found in Table 1 where I refer to the cell current, as opposed to the local current.

Table 1: Differences between a homogeneous and the multibunch model

\begin{tabular}{|c|c|c|}
\hline Parameter & Homogeneous & Multibunch, order $n$ \\
\hline OCV & $U\left(Q_{\text {cell }}\right)$ & $U\left(Q_{i}\right), i=[1 . . n]$ \\
\hline$R_{H F}$ & $R_{e}(S O C, I, T)+$ & $R_{H F, i}(I, T)$ \\
& $R_{c t}(S O C, I, T)$ & and distributed \\
\hline$R_{d}$ & $R_{d}(S O C, I, T)$ & $R_{d}(S O C 50 \%, I, T) \cdot n$ \\
\hline$C_{d}$ & $C_{d}(S O C, I, T)$ & $C_{d}(S O C 50 \%, I, T) / n$ \\
\hline
\end{tabular}

Regarding the number of bunches $n$, several configurations have been tested, and the optimal number found for a single cell seems to be the higher one. The higher the number of bunches, the more precise the model is. Because the optimization time needed to find a set of resistance is roughly a first order function of the number of bunch, the chosen number here is a compromise between accuracy and computation time. The impact of $n$ on accuracy and computation time for a given method can be found on figure 3. The result regarding the value of $n$ on accuracy and computation time for other methods are similar and are not presented here. All used methods are presented in section 3.2. For this study, we choose $n=20$.

On figure 4 are displayed the voltages simulated for a various number of bunches for a given distribution of parameter. The error decrease with the increase of bunch number for multiple reasons. First of all, due to the fact that a SoC modification does not affect the parameter's value, the over-voltage is only a function of the current, which is constant here, and of the temperature, which is monotonically increasing. So in a first approximation, one can estimate that the over-voltage is a function of the temperature only. As a temperature increase lead to a decrease in resistance value, the over-voltage for one bunch is a decreasing function of the temperature, thus of the time. That is why the shape of the OCV, and especially the transition between the plateaus are visible on the 1-bunch voltage. This lead to a great error in the end of the discharge. 


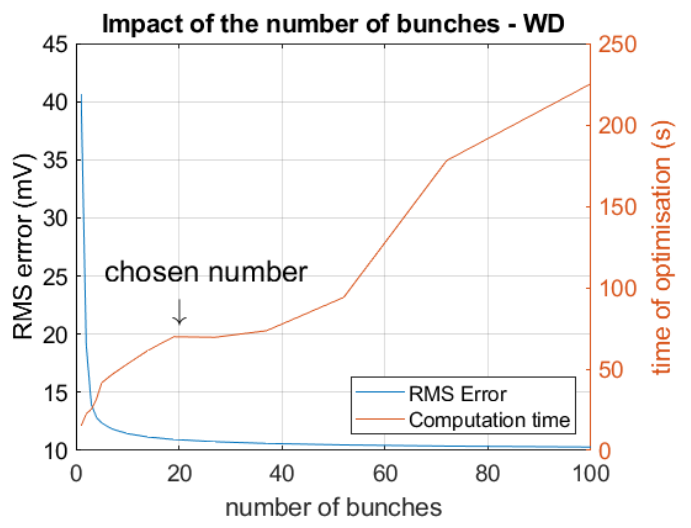

Figure 3: Influence of the number of bunches

Usual homogeneous model uses a SoC-dependency lookup-table to tackle this problem, with an increase in the resistance at low and high $\mathrm{SoC}$ (the effect is the same during a charge, where the over-voltage is usually quickly increasing near the end of the discharge, this is why the constant voltage protocol is so widely used). In this article, the SoC-dependency is not modelled by choice. As one can see on figure 5, increasing the number of bunches give a satisfactory result regarding the voltage drop in the end of the discharge, as the least resistive bunches begin to be completely discharged, letting more current flow through high resistances one, effectively increasing their over-voltage, and lowering the equivalent voltage of the whole model.

Another effect of the increase of the number of bunches is the disappearance of the transitions between the plateaus. This lead to a smoother curve, which is in accordance with the measurement one can get from a high-current battery discharge. This is due to the fact that the transition between the OCV plateau does not occur at the same time for all the bunches, as the least resistive bunches are discharged quicker. This phenomenon "hide" the plateaus transition by smoothing them over a larger SoC range. As a result, for an increasing number of bunches, the error decrease, until a certain point where an augmentation of the number of bunches do not significantly decrease the error, and where the computation time becomes too large for the achieved precision compared to other choices of number of bunches, as depicted on figure 3 .

\section{Simulation and optimization algorithms}

\subsection{Simulation of the cell}

Based on a homogeneous bunch model where the parameter and non-linearity tables are known, we build the multibunch model by determining the IRD. That is, we need to find a set of resistances $R_{H F, i}$ that allows the model to simulate the battery for any discharge inside the operating conditions. This is done by using an optimization in order to find the best set of $R_{H F, i}$. The flow chart of our coupled simulation-optimization algorithm is represented on figure 6 .

The voltage is simulated thanks to several inputs: the measured temperature and current during a discharge, the initial state of the cell and the IRD. The simulation algorithm has three steps.

- First the electrical parameters values (resistances, capacities, time constants) are updated with regard to current and temperature (Equation (2));

- Then, based on the previous bunch currents and the assumption that they remain constant during a time step, the local states of charge $Q_{i}$ and voltages $U\left(Q_{i}\right)$ and the voltage across the RC element are computed (Equation (1));

- Finally, the new bunch currents and cell voltage are computed by solving a linear system using the cell current, the locals OCV $U\left(Q_{i}\right)$ and the voltages across the electrical parameters.

\subsection{Optimization algorithm and methods}

In order to simplify the definition of the optimization function, we chose to use high frequency conductances $G_{H F, i}$ instead of high frequency resistances $R_{H F, i}$. 


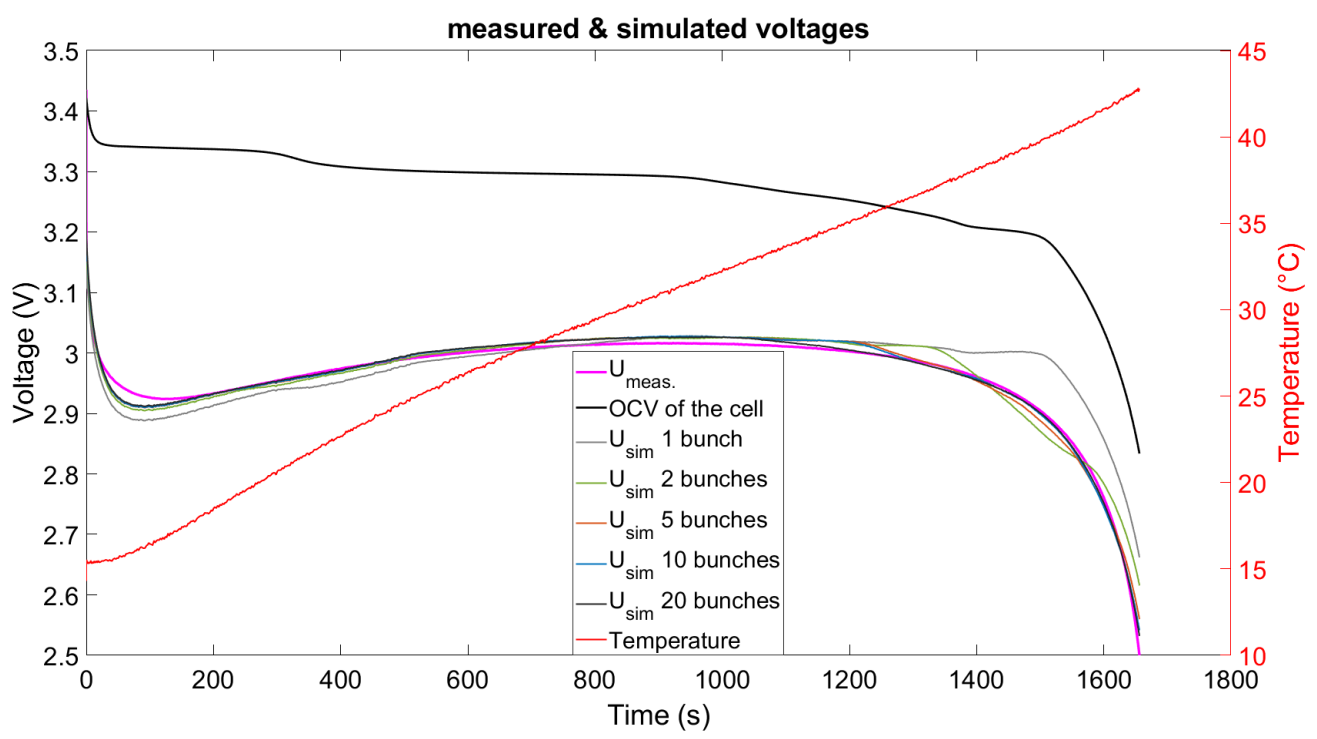

Figure 4: Influence of the number of bunches on the simulated voltage

This is because the equivalent conductance of parallel conductances is simply the sum of those conductances.

This section is dedicated to the determination of the $R_{H F}$ values, that are distributed among the bunches. To find the best set, three methods are compared:

- WD method: an indirect determination using a Weibull Distribution;

- FD-WI method: a direct determination using a Free Distribution with a Weibull Initialization;

- FD method: a direct determination using a Free Distribution initialized by a uniform one between two values.

A Weibull distribution is a mathematical distribution law that allows a great variety of shape, expected value and initial value. It's probability density function is

$$
f(x ; \lambda, k, \theta)=\frac{k}{\lambda}\left(\frac{x-\theta}{\lambda}\right)^{k-1} \exp ^{-\left(\frac{x-\theta}{\lambda}\right)^{k}}
$$

Using the WD method was already done in a previous article Damay et al. (2017). Here we propose, on the one hand, an optimization of the three Weibull parameters of the indirect determination and, on the other hand, the possibility to directly find the $R_{H F, i}$ values that were previously extracted from the WD. As the aim of the IRD is to be monitored in order to make a diagnosis of the cell, a shape modification of the IRD must remain possible. Thus a Weibull distribution is not the best suited one as we could miss an outsider in the IRD during the life of the cell. Using a free distribution allows us to be able to find those points, at the cost of increasing the number of parameters, therefore the computation time, to the number of bunches $n=20$. In order to decrease the computation time, we imposed an ordered solution, meaning that an initial conductance $G_{1}$ is searched for and then the difference between $G_{n}$ and $G_{n+1}$ (equation (4))

$$
\forall i, j \in[2, n], \quad G_{H F, i}=G_{H F, 1}+\sum_{j=2}^{i} \Delta G_{j}
$$

subject to $\quad \forall j \in[2, n], \quad 0 \leq \Delta G_{j} \leq \Delta G_{\max }$

This way of ordering our solution was necessary as the algorithm used in the software Matlab includes the optimization function lsqnonlin which does not have constraint options in order to sort the solution. Only lower and upper bounds can be defined, which goes well with a research of the differences, as the lower bound can be defined to be strictly superior to 0 , forcing an increase from one value to another.

The objective function for a free distribution is then:

$\min _{G_{H F, i}} f_{e}\left(t, I, T, G_{H F, i}\right)=\left[U_{\text {sim }}\left(t, I, T, G_{H F, i}\right)-U_{\text {meas }}(t)\right]$

where $f_{e}$ is the error vector for a given discharge.

The objective function to be minimized is the difference between the simulated voltage and a measured 


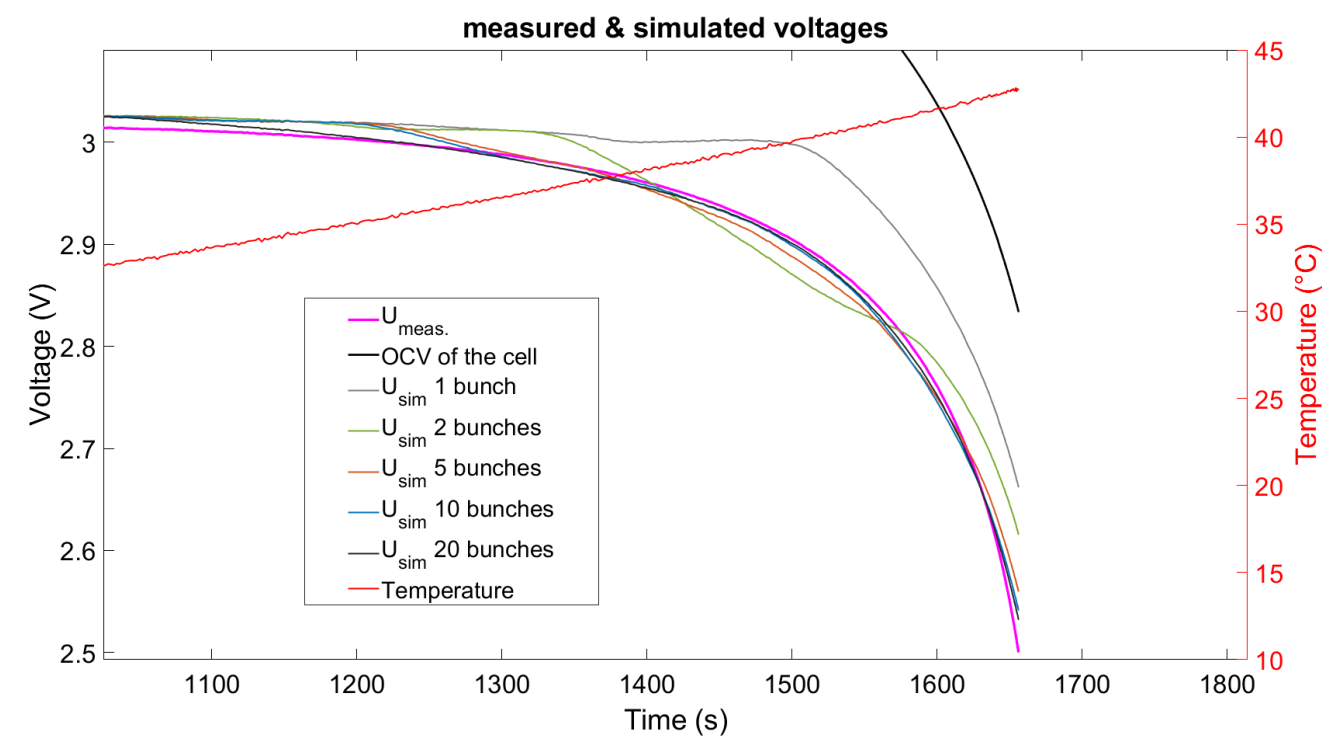

Figure 5: Zoom on the end of the discharge

voltage. Thus, we need a test used as optimization test in order to determine our IRD. This test, the setup and the cells used are presented in the next section.

\section{Experimental conditions}

We used two 40Ah $\mathrm{LiFePO}_{4} /$ graphite cells from the same batch for this study. The discharge current limit is $2 \mathrm{C}$, the charge current limit is $1 \mathrm{C}$, for a voltage varying between 2,5 and 3,7V. The operating-temperature range is -15 to $50^{\circ} \mathrm{C}$. The first cell used is already aged and its actual capacity is around $37.9 \mathrm{Ah}$. This cell was kept at room temperature for 2 years and various tests have been made to acquire data for different studies. No particular aging plan was used to age this cell. The second cell used for this article is a cell from the same batch, that was kept at $5^{\circ} \mathrm{C}$, and which capacity is around 39.4Ah.

The first cell was used to make several discharges in various conditions in order to determine its IRD and to run several validation simulations in order to validate the behaviour of the model when used in different operating conditions. The same method has been used to determine the IRD of the second cell in order to compare it with the IRD of the first cell and discuss the applicability of the proposed IRD determination method for diagnosis purpose.

\subsection{Experimental setup}

As explained in section 2.2, the parameters values are varying with regards to the temperature. It is measured on the centre of the largest face of the battery by using a thermocouple. But as we explained previously, our model is not a spatial one, as no assumptions are made regarding the position of the elementary volumes that are grouped inside a given bunch. Thus the temperature to be taken into account for a bunch may be different from the measured surface temperature. Thus, as the measured temperature will be used to compute the parameters values of all bunches, the cell needs to be as homogeneous in temperature as possible. We chose to thermally insulate the cell with a $10-\mathrm{cm}$ thick polyurethane box during all our tests, as pictured on figure 7 , in order to have a quasi-adiabatic setup. We used glass wool on the top of the cell to be able to connect the power wires. The latter are also insulated with polyurethane foam. The whole setup was then placed into a climatic chamber to control the initial temperature of the cell and to connect it to a Bio-Logic system with a $100 \mathrm{~A}$ booster.

We then consider the temperature to be the same in the surface and inside the battery. Support to this assumption can be found in the article of Damay et al. Damay et al. (2015), in which the thermal exchange between the core of the battery and the ambient air, through the largest face of the cell, has been modelled. The experimental setup was the same except that the 


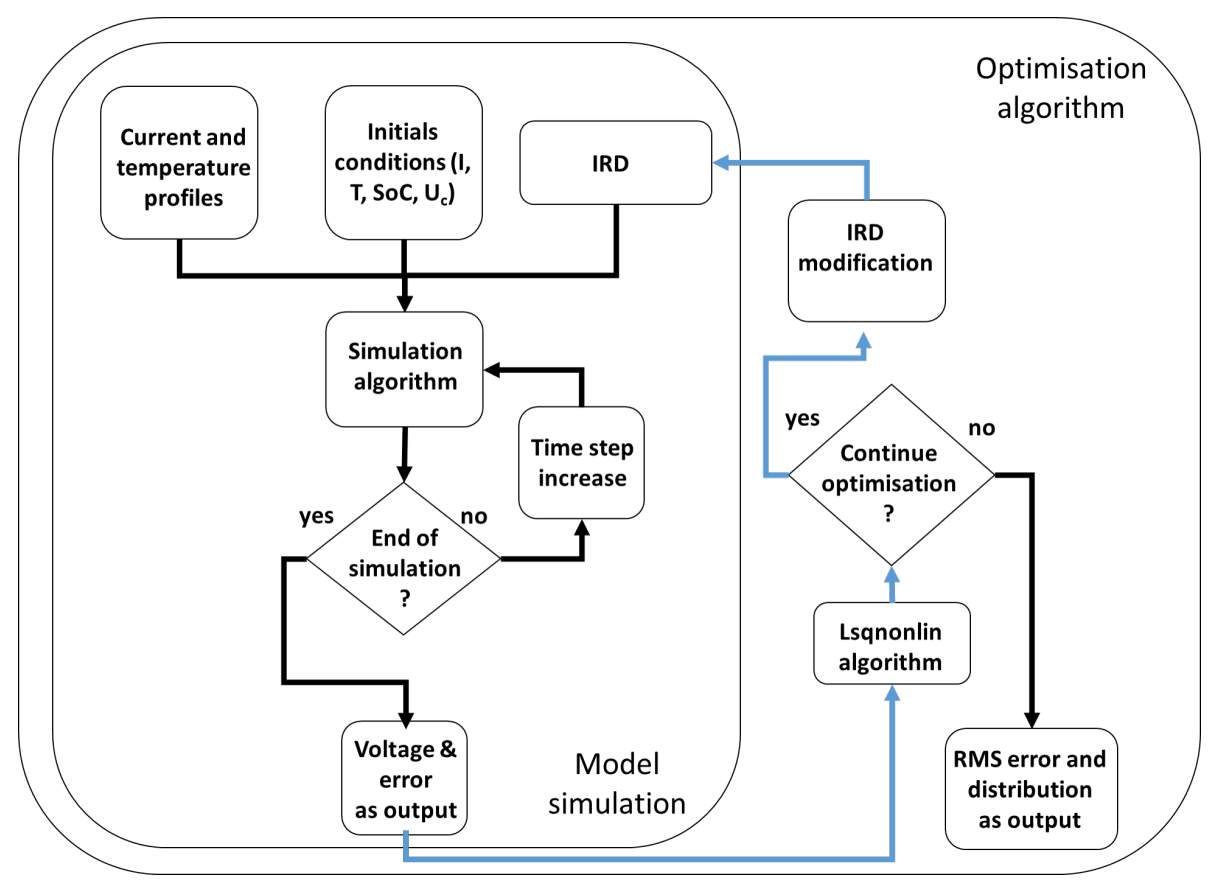

Figure 6: flow chart of our algorithm

bottom face was cooled by a thermally regulated plate with thermal glue. The thermal resistance between the core and the largest face of the battery $R_{i n}$ has been estimated to be of $0.8 \mathrm{~K} / \mathrm{W}$. The thermal resistance between the largest face and the ambient air $R_{\text {out }}$ has been estimated to be of $141 \mathrm{~K} / \mathrm{W}$. As $R_{\text {in }}$ is very small compared to $R_{\text {out }}$, we assume that the temperature difference between the largest face and the core of the cell is very small compared to the temperature difference between the ambient air temperature and the largest face temperature.

In the same article, a 3 dimensional thermal model was used to simulate the core temperature of the cell during a full discharge at $2 \mathrm{C}$ with an initial temperature of $18^{\circ} \mathrm{C}$. The difference between the estimated core temperature and the surface temperature is less than $3^{\circ} \mathrm{C}$ when the surface temperature reaches $40^{\circ} \mathrm{C}$ and less than $1^{\circ} \mathrm{C}$ when the surface temperature reaches $25^{\circ} \mathrm{C}$. As the cell is cooled by the bottom, effectively creating gradient of temperature inside the cell, thus this can be viewed as "worst case" scenario. This small difference in temperature is due to the fact that the internal heat resistance is very low, thus ensuring a good homogeneity of the temperature inside the cell. Considering a thermal difference of $3^{\circ} \mathrm{C}$ between the core and the surface of the cell leads to an error of the estimated electrical parameters used in this article of 8 to $20 \%$ for the high

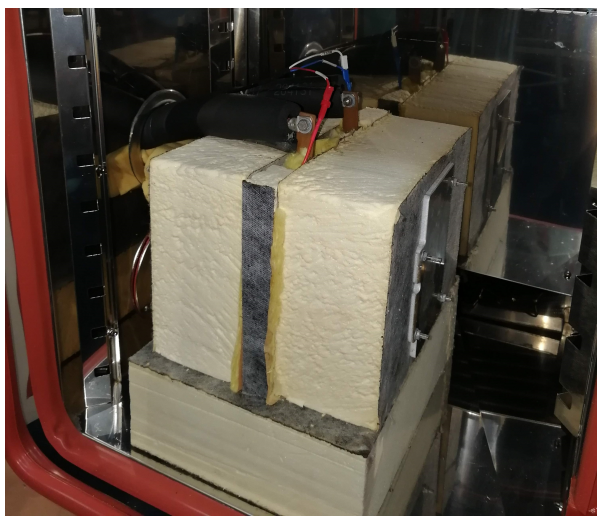

Figure 7: Experimental setup

frequency resistance, of 8 to $11 \%$ for the diffusion resistance and of 1.6 to $9 \%$ for the diffusion capacity.

\subsection{Tests descriptions}

The IRD determination test and the validation tests are constant current discharge from the higher cut-off voltage to the lower cut-off voltage. The IRD determination test consists in a discharge at low initial temperature $\left(15^{\circ} \mathrm{C}\right)$ and high current $(2 \mathrm{C}$, that is $80 \mathrm{~A})$. The validation tests are similar but with an initial temperature of $25^{\circ} \mathrm{C}$ and $38^{\circ} \mathrm{C}$ with a current of $1 \mathrm{C}$ and $2 \mathrm{C}$ for each temperature, as well as a test at $15{ }^{\circ} \mathrm{C}$, with a current 
Table 2: Experimental conditions of the tests

\begin{tabular}{|c|c|c|}
\hline $\begin{array}{c}\text { Current } \rightarrow \\
\text { Initial temp. } \downarrow\end{array}$ & $1 \mathrm{C} \mathrm{(40A)}$ & $2 \mathrm{C}(80 \mathrm{~A})$ \\
\hline $15^{\circ} \mathrm{C}$ & $\begin{array}{c}\text { Validation } \\
\text { test }\end{array}$ & $\begin{array}{c}\text { IRD determination } \\
\text { test }\end{array}$ \\
\hline $25^{\circ} \mathrm{C}$ & $\begin{array}{c}\text { Validation test } \\
\text { test }\end{array}$ & $\begin{array}{c}\text { Validation } \\
\text { test }\end{array}$ \\
\hline $38^{\circ} \mathrm{C}$ & $\begin{array}{c}\text { Validation test } \\
\text { test }\end{array}$ & $\begin{array}{c}\text { Aborted validation } \\
\text { test }\end{array}$ \\
\hline
\end{tabular}

of 1C. The maximum temperature allowed by the constructor has been hit during the $2 \mathrm{C}$ discharge with initial temperature of $38^{\circ} \mathrm{C}$, resulting in a partial discharge only. This discharge is not exploited here and no simulation have been made using experimental condition. The table 2 contains the different operating conditions and the associated tests.

\section{Results and discussions}

\subsection{Choice of the IRD determination test}

Our model aims to model the heterogeneity of a single cell. Thus a discharge that brings out the most heterogeneous behaviour of the cell should be the best suited one in order to determine the IRD of our model. Once this IRD is determined, it will be used to simulate other operating conditions with a comparison to experimental measurements to determine if it can model the cell in a wide range of operating conditions. Based on our observations, the discharge with the most heterogeneous behaviour is a low temperature - high current one. We tried to determine IRD with other operating conditions, such as higher temperature or lower current, but it gave greater error when the IRD is applied to the other conditions of simulation. Thus this particular discharge at low temperature and high current was kept as determination test for the IRD.

The low temperature brings more dispersed impedance values as temperature is a major factor for impedance variation. The high current brings state of charge heterogeneity among parts of the cell, because our cell has a flat OCV as a function of the SoC. This lead to no electrochemical constraint between a low SoC and a mid to high SoC local part of the cell. Moreover, as the setup only is a quasi-adiabatic setup, the temperature increases in the case of a high current should be greater than with a lower current, effectively covering a larger temperature range. This will ensure our model robustness with respect to large temperature variations.

We used our IRD determination algorithm (figure 6), tests (see section 4.2) and methods (see section 3.2) to determine all three IRDs. The result of the indirect WD method, that was developed in a previous study Damay et al. (2017), are compared on figure 8 with the results of the direct FD-WI method that we propose in this paper.

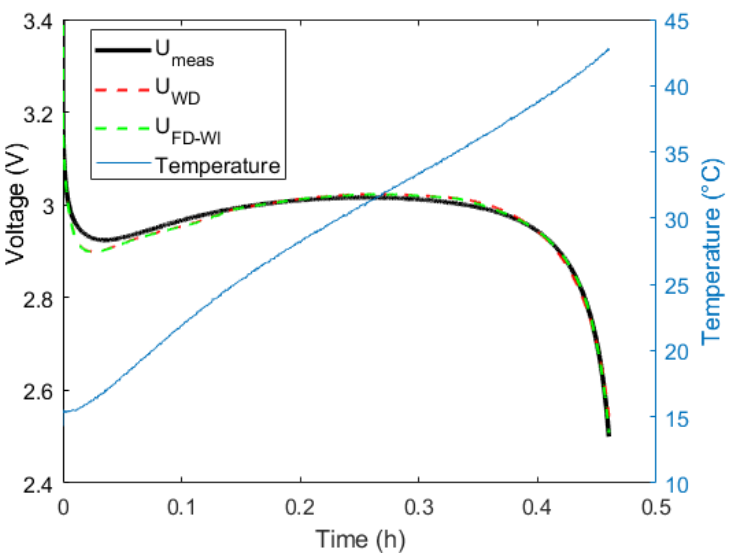

(a) Overall measurements and simulations

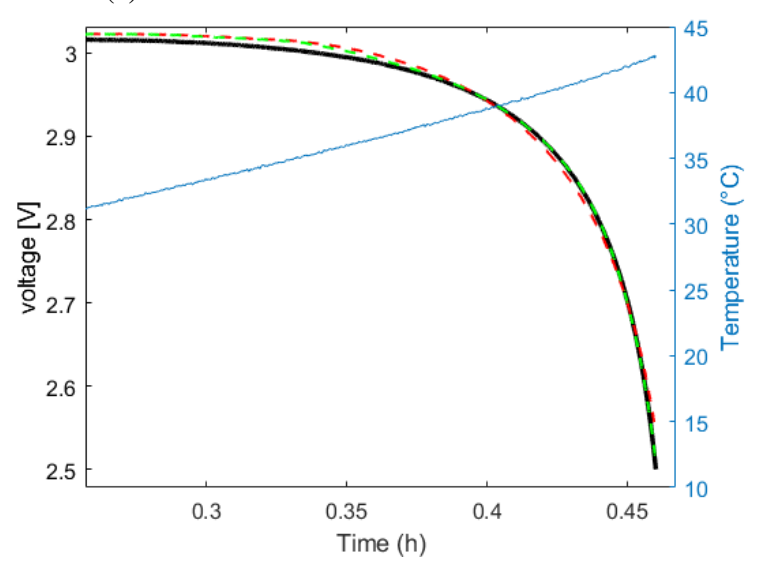

(b) Zoom on the end of the discharge

Figure 8: Measured temperature, voltage and simulated voltages

From an initial temperature of $15^{\circ} \mathrm{C}$, we observe an increase up to $43{ }^{\circ} \mathrm{C}$. The linear shape of the temperature curve supports the quasi-adiabatic setup that we did, as a thermal cooling from the cell would be detected as a variation of the derivative of the temperature curve.

As the impedance is strongly linked to temperature in a cell, we observe a particular shape of the voltage curve. In a first part a voltage drop due to ohmic resis- 
tance can be seen. Then the voltage increases as the state of charge decrease. As the cell is an LFP/graphite cell, the OCV is nearly-flat at the beginning of the discharge, which should induce a flat voltage during discharge. However, the rising temperature of the cell leads to a decrease of its impedance and, thus, leads to an increase of the cell voltage. This happens until the OCV of the cell decreases at low SoC, effectively decreasing the cell voltage.

\subsection{Relevance of the determined IRD to a diagnosis purpose}

The aim of the optimization is to find a suitable set of resistances to model the cell. However, we also want to obtain a distribution that is physically meaningful. Because the goal of the IRD is to monitor the internal state of the cell, we have to exclude the results from the WD method from the acceptable results, as it is so far not proved that the resistances inside a cell vary with respect to a Weibull distribution. Besides, a Weibull distribution is constrained by equation (3) and cannot represent all possible IRD that a cell may have.

We found that the FD method may lead to a questionable solution. On figure 9 are presented the distributions that our algorithm finds for each method presented in section 3.2. The FD method brings a distribution with a few equals $\&$ subsequent values and one greater value at the end. Such an evolution is possible but is considered less likely because it doesn't suit the hypothesis of a continuum of electrical properties inside the cell. The FD-WI method brings a better regularity among the IRD, as expected from the model construction.

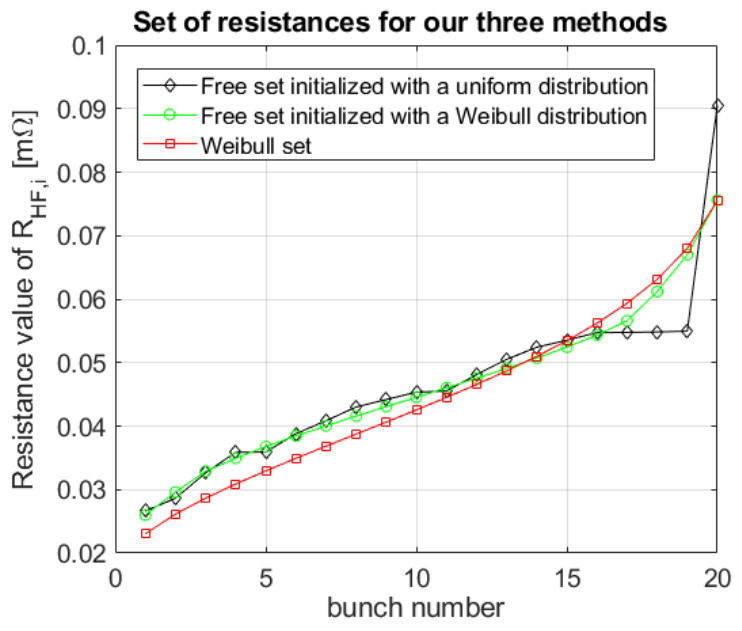

Figure 9: Comparison of the IRD
As we find it hard to have a consistent criterion to evaluate the qualities of those solutions, we recommend the FD-WI method for the determination of the IRD as it gives satisfactory results regarding the hypothesis of an internal electrical-properties continuum. It has the advantage to be quickly initialized by the WD method in which only three parameters have to be optimized, and to avoid purely mathematical IRD.

\subsection{Validation tests}

To verify that the determined IRD is consistent, we simulated discharges for other operating conditions. The results are presented on figure 10. On each subfigure are displayed the measured voltage and the simulated voltage for the indicated operating condition. The results validate our IRD determination method as the error is kept below $25 \mathrm{mV}$ for an average voltage around $3.1 \mathrm{~V}$ (that is to say, less than $1 \%$ relative error). The various operating points that are simulated strongly support the fact that our model has a physical meaning, that can be used to track the IRD during the cell lifetime.

The different simulations show a smaller error for a higher discharge current. We suppose that high currents are more likely to induce a heterogeneous behaviour inside the cell, which are to be modelled by this heterogeneous model. Although the determination test was also a high current test, which can lead to a bias in the error, IRD extracted from low-current test did not give better result, neither for high current, nor for low ones, as specified in section 5.1. Thus this IRD is considered to be the best one we could find. The overall shape of the simulation is satisfying compared to measured voltage, which validate our goal to simulate the cell voltage over a wide range of operating points.

As the cell voltage can be simulated during a constant current discharge, with only one IRD obtained by optimization on a single discharge at low temperature and high current, it becomes possible to track the evolution of this IRD. This open the way to a non-invasive diagnosis tool for a single cell or a group of parallel cells.

\subsection{Direct IRD determination as a diagnosis tool}

After having determined that the proposed IRD determination method is suitable for a diagnosis purpose, we tested this method in its usage as a diagnosis tool to compare the IRD of two cells that have different ageing conditions (those cells were described in section 4). We expect the distribution of the aged cell to show higher values of resistances, as its resistance should have increased with use. 


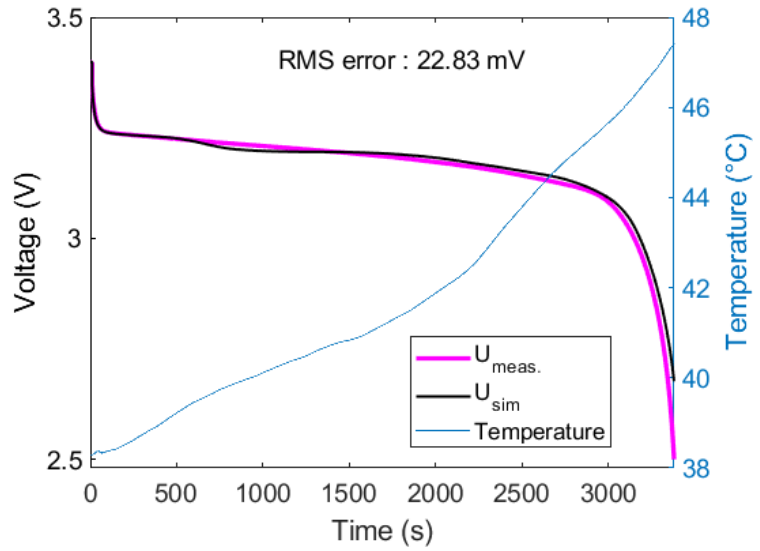

(a) $1 \mathrm{C}$ discharge, $T_{\text {ini }} 38^{\circ} \mathrm{C}$

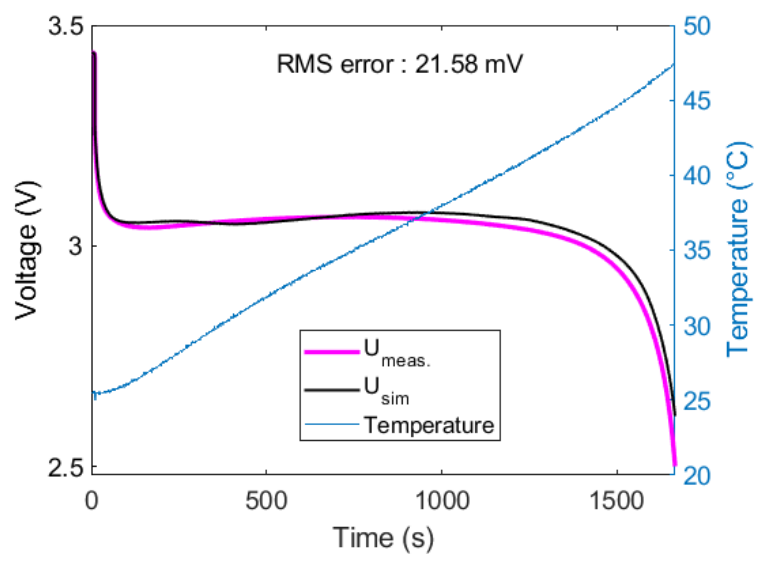

(c) $2 \mathrm{C}$ discharge, $T_{\text {ini }} 25^{\circ} \mathrm{C}$

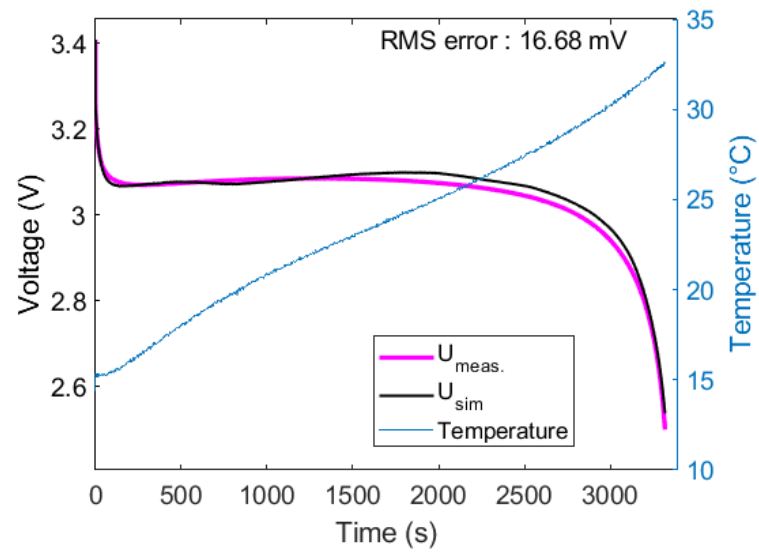

(b) $1 \mathrm{C}$ discharge, $T_{\text {ini }} 15^{\circ} \mathrm{C}$

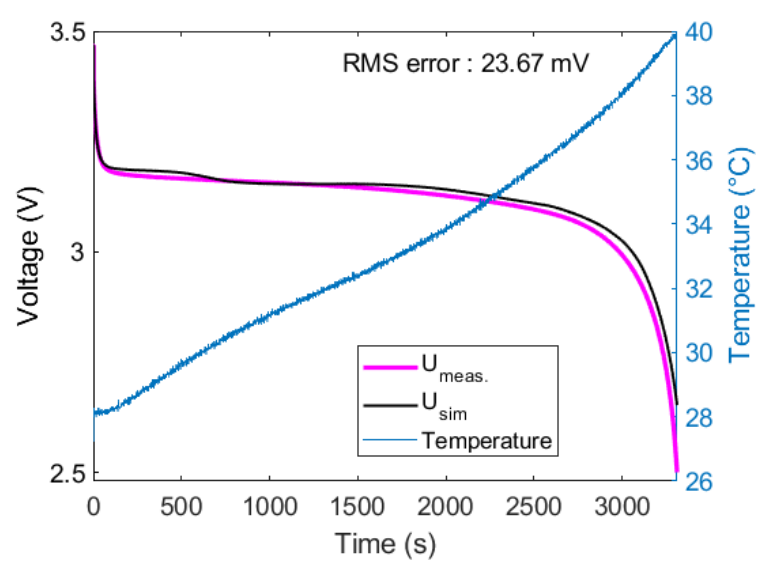

(d) $1 \mathrm{C}$ discharge, $T_{i n i} 25^{\circ} \mathrm{C}$

Figure 10: Measured temperature, voltage and simulated voltage for different operating conditions

The exact same multibunch model parameters (and non-linearity regarding temperature and current) have been used for the new cell, except the values of $R_{H F}$. Those values were determined with the IRD determination test. The discharge test was conducted with the same protocol, and in the same operating conditions. The results of the optimization of the resistances are shown on figure 11.

The IRD values are displayed on the $\mathrm{Y}$ axis and the number of the bunch on the $\mathrm{X}$ axis. Both cells show very similar shape of IRD, with the aged battery having a higher equivalent resistance value as expected. The values of the aged cell resistances are a bit higher by around $4 m \Omega$ for bunches 4 to 14 but the shape is preserved, as if the aging impacted bunches 1 to 18 homogeneously. The bunches 18 to 20 are interesting as we see a crossover between the IRD. This can be due to several causes, including
- a difference between the two batteries initial IRD;

- an unexpected degradation process;

- a difficulty in finding a global optimum as local minima may exist (equation (5));

- an artefact due to the model construction hypothesis (very simple bunch model, no separation of the 2 electrodes behaviours...).

So far, no evidence has been found toward any of those causes, and further studies should help us to have a better understanding of these results. Tracking a single cell IRD during its lifetime would help to distinguish that, as its resistance should only grow.

The IRD analysis shows good consistency with the expected resistance values, as the aged cell has a higher average value of equivalent resistance. More tests on other types of battery are necessary to conclude about 


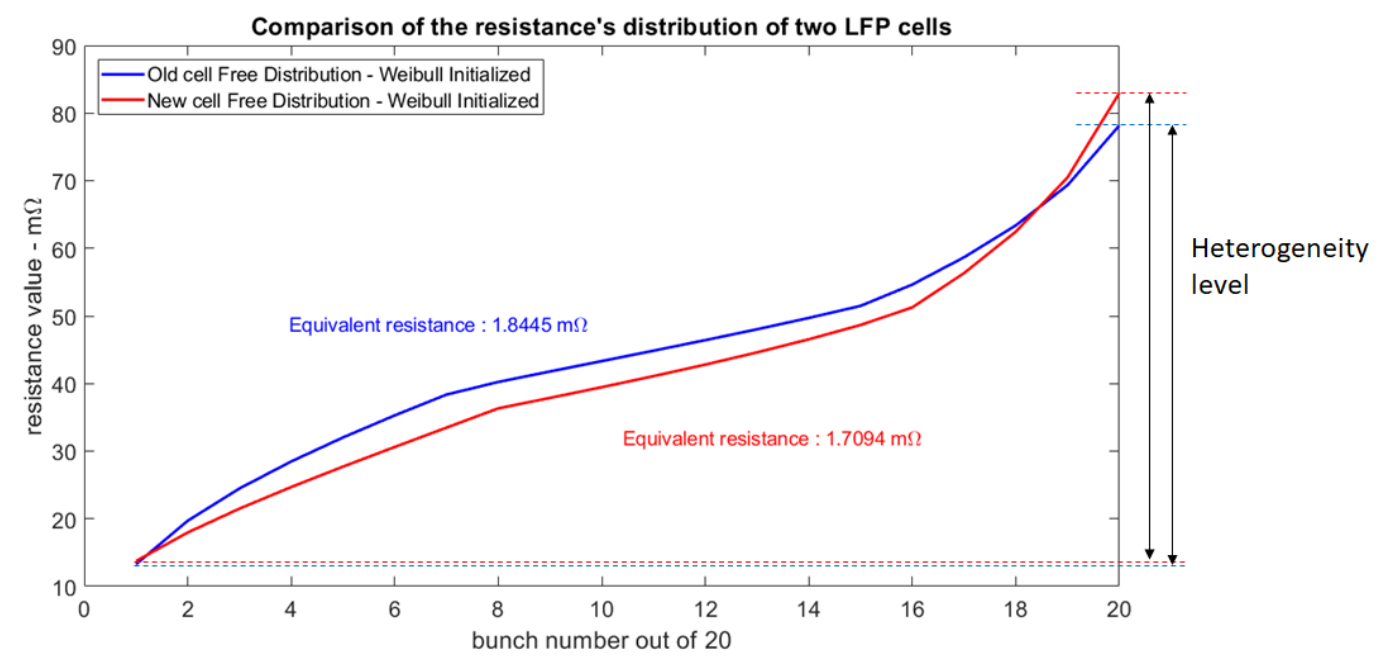

Figure 11: Comparison of the IRD for an aged and a new cell

the usage of this criterion as a $\mathrm{SoH}$ diagnosis tool, but those first results are encouraging. Moreover, new tool can be used, based on the IRD shape or values, such as a heterogeneity level that is the difference between the min value of the IRD and the max value of the IRD. As the cell ages normally, we expect this ratio to decrease as less resistive parts are privileged paths for the current, inducing more degradations and a resistance increase. As this tool can be used on aged cell without a need to rebuild non-linearity tables, it makes a good candidate for a SoH diagnosis tool. Other criteria such as the standard deviation of the IRD could be used and will be investigated in other studies. This work may bring new methods in order to diagnose the internal behaviour of the cell, without opening them.

\section{Conclusions and perspectives}

In this paper we presented an equivalent circuit model called multibunch model, whose goal is to model the heterogeneity of a commercial $\mathrm{LiFePO}_{4} /$ graphite cell. This model was initially developed in a previous study Damay et al. (2017) and, in this article, we demonstrate that it can be used as a non-invasive diagnosis tool. This model gives access to an internal resistance distribution (IRD) which is assumed to represent the distribution of the cell internal electrical properties.

We propose a direct determination method of the IRD in this paper, as opposed to the indirect determination method of the previous study. This allows us to have a responsive distribution that is not bound to a mathematical law of a distribution. As the cell internal properties may vary in many different ways, the ability to spot outsiders values within the IRD is a strong improvement of the method.

An experimental protocol has been proposed for the IRD determination and three determination methods have been compared. The first one is the previous study indirect determination method using a Weibull distribution. The two others are a direct determination using a free optimization of the IRD values, after initializing them with either a Weibull distribution or a uniform distribution. We demonstrated that the best method is to first determine the best Weibull distribution, then to allow an optimization of the internal resistances around the Weibull values in order to fit the measurements obtained thanks to the IRD determination protocol. The found IRD was validated for different other operating conditions with an error of less than $25 \mathrm{mV}$ for an average voltage of $3.1 \mathrm{~V}$.

The comparison of the IRD of two different cells highlights the new insights that our method provides about a single cell internal state. We propose an "heterogeneity level" as a new criterion to determine a cell state of health, which allows to have a better accuracy on the cell internal resistance. Other criteria could be found to better represent the cell ageing with regard to the IRD information, but further study and tests on other batteries are necessary to have a motivated opinion on the subject. This is the next step of our work and will be presented in future publications.

The use of thermal model to estimate the core tem- 
perature, and the correct the estimation of the electrical parameter may be an interesting perspective of this study. Especially when low errors are achieved and where small variations of the parameters due to temperature may account for an unneglectable part of the remaining error.

Also, application of this method to a small battery pack which contains one aged cell and several new cells will be done in order to validate this method as a diagnosis tool for larger battery pack. The construction of the multibunch model allows it to model several batteries in parallel. As our heterogeneous model is made of parallel bunches, to model parallel cells is easy to parametrize, as we only need to increase the number of bunches.

Finally, we plan to apply this simulation method to other cell chemistries to verify that we are able to simulate them over their whole operating range, to get an insight of their internal states, and to follow the evolution of their IRD in order to have a non-invasive diagnosis tool for large battery packs.

\section{Acknowledgements}

This work was funded by the French railway company SNCF as part of a CIFRE Thesis between SNCF, Université de technologie de Compiègne and the ANRT (National Agency for Research and Technology).

\section{References}

Andre, D., Meiler, M., Steiner, K., Walz, H., Soczka-Guth, T., Sauer, D.U., 2011. Characterization of high-power lithium-ion batteries by electrochemical impedance spectroscopy. II: Modelling. Journal of Power Sources 196, 5349-5356.

Bernardi, D.M., Go, J.Y., 2011. Analysis of pulse and relaxation behavior in lithium-ion batteries. Journal of Power Sources 196, 412427.

Berrueta, A., Urtasun, A., Ursúa, A., Sanchis, P., 2018. A comprehensive model for lithium-ion batteries: From the physical principles to an electrical model. Energy 144, 286-300.

Cooper, S.J., Eastwood, D.S., Gelb, J., Damblanc, G., Brett, D.J., Bradley, R.S., Withers, P.J., Lee, P.D., Marquis, A.J., Brandon, N.P., Shearing, P.R., 2014. Image based modelling of microstructural heterogeneity in LiFePO 4 electrodes for Li-ion batteries. Journal of Power Sources 247, 1033-1039.

Damay, N., Forgez, C., Bichat, M.P., Friedrich, G., 2015. Thermal modeling of large prismatic LiFePO4/graphite battery. Coupled thermal and heat generation models for characterization and simulation. Journal of Power Sources 283, 37-45.

Damay, N., Forgez, C., Friedrich, G., Bichat, M.P., 2017. Heterogeneous behavior modeling of a LiFePO 4 -graphite cell using an equivalent electrical circuit. Journal of Energy Storage 12, 167177.

Delacourt, C., 2016. Analyse des Performances et du Vieillissement des Batteries à Ions Lithium par des Méthodes de Modélisation Mathématique .
Di Domenico, D., Stefanopoulou, A., Fiengo, G., 2010. LithiumIon Battery State of Charge and Critical Surface Charge Estimation Using an Electrochemical Model-Based Extended Kalman Filter. Journal of Dynamic Systems, Measurement, and Control 132, 061302.

Dong, T., Peng, P., Jiang, F., 2018. Numerical modeling and analysis of the thermal behavior of NCM lithium-ion batteries subjected to very high C-rate discharge/charge operations. International Journal of Heat and Mass Transfer 117, 261-272.

Kuhn, E., Forgez, C., Lagonotte, P., Friedrich, G., 2006. Modelling $\mathrm{Ni}-\mathrm{mH}$ battery using Cauer and Foster structures. Journal of Power Sources 158, 1490-1497.

Merla, Y., Wu, B., Yu, V., Martinez-botas, R.F., Gregory, J.O., 2018. An easy-to-parameterise physics-informed battery model and its application towards lithium-ion battery cell design, diagnosis, and degradation 384, 66-79.

Nuhic, A., Terzimehic, T., Soczka-Guth, T., Buchholz, M., Dietmayer, K., 2013. Health diagnosis and remaining useful life prognostics of lithium-ion batteries using data-driven methods. Journal of Power Sources 239, 680-688.

Ouvrard, G., Zerrouki, M., Soudan, P., Lestriez, B., Masquelier, C., Morcrette, M., Hamelet, S., Belin, S., Flank, A.M., Baudelet, F., 2013. Heterogeneous behaviour of the lithium battery composite electrode LiFePO4. Journal of Power Sources 229, 16-21.

Park, S.Y., Baek, W.J., Lee, S.Y., Seo, J.A., Kang, Y.S., Koh, M., Kim, S.H., 2018. Probing electrical degradation of cathode materials for lithium-ion batteries with nanoscale resolution. Nano Energy 49, $1-6$.

Saha, B., Goebel, K., Poll, S., Christophersen, J., 2009. Prognostics Methods for Battery Health Monitoring Using a Bayesian Framework. Instrumentation and Measurement, IEEE Transactions on 58, 291-296.

Satyavani, T.V., Ramya Kiran, B., Rajesh Kumar, V., Srinivas Kumar, A., Naidu, S.V., 2016. Effect of particle size on dc conductivity, activation energy and diffusion coefficient of lithium iron phosphate in Li-ion cells. Engineering Science and Technology, an International Journal 19, 40-44.

Schuster, S.F., Brand, M.J., Berg, P., Gleissenberger, M., Jossen, A., 2015. Lithium-ion cell-to-cell variation during battery electric vehicle operation. Journal of Power Sources 297, 242-251.

Yuan, S., Jiang, L., Yin, C., Wu, H., Zhang, X., 2017. A transfer function type of simplified electrochemical model with modified boundary conditions and Padé approximation for Li-ion battery: Part 1. lithium concentration estimation. Journal of Power Sources 352, 245-257.

Zhao, W., Luo, G., Wang, C.Y., 2014. Effect of tab design on largeformat $\mathrm{Li}$-ion cell performance. Journal of Power Sources 257, 70-79. 